\section{Hochfrequenzdiathermie - ein neues Verfahren in der Therapie maligner und benigner Atemwegsstenosen}

Zusammenfassung: Die Hochfrequenzdiathermie ist ein relativ neues Verfahren zur Behandlung maligner oder benigner Atemwegsstenosen. Wir berichten über die Ergebnisse von 58 Eingriffen bei insgesamt 41 Patienten (maligne Stenosen $n=30$, benigne Stenosen $n=11$ ) in einem Zeitraum von drei Jahren. Zur Verfügung standen verschiedene Diathermieinstrumente (Kontaktsonde, Messer mit 4, 5 und $7 \mathrm{~mm}$ Länge, Zange und Schlinge). 53/58 Sitzungen fanden in Allgemeinnarkose statt, die Energie wurde stets auf $40 \mathrm{~W}$ begrenzt bei unbegrenzter Pulsdauer. Die am häufigsten (49/58) verwendeten Instrumente waren die Diathermiemesser mit einer bevorzugten Länge $5 \mathrm{~mm}$, wobei die Instrumente entweder zum präzisen Abtrennen von Tumor- oder Narbengewebe oder zur direkten Koagulation eingesetzt werden konnten. Der Einsatz der Kontaktsonden oder Zangen war hingegen regelmäßig erschwert durch eine rasche Bildung einer Detritusschicht um das Instrument. Gestielte Polypen, die am einfachsten mit der Schlinge abzutragen waren, wurden nur bei drei Patienten gefunden. Bei zwei Patienten ereignete sich eine größere Blutung $(>100 \mathrm{ml})$. In keinem einzigen Fall kam es nach den Diathermieeingriffen zur Verlegung durch Fibrinmembranen. Zusammenfassend kann die Hochfrequenzdiathermie als eine effektive und sichere Methode zur endobronchialen Resektion angesehen werden und stellt eine gute Alternative zum Laser als dem klassischen Verfahren dar.

Schlüsselwörter: Hochfrequenzdiathermie - Bronchoskopie Atemwegsstenose - Bronchialkarzinom

High Frequency Electrocautery - A New Method in the Treatment of Malignant and Benign Stenoses of the Airways: High-frequency electrocautery is a relatively new method in the treatment of malignant or benign airway stenoses. We report on the results of 58 sessions in 41 patients (malignant condition $n=30$, benign $n=11$ ) within a three-year period. Various instruments were available for coagulation (blunt probe, knives of 4, 5 and $7 \mathrm{~mm}$ length, forceps and wire snare). 53/ 58 sessions were performed under general anaesthesia, energy was limited to $40 \mathrm{~W}$ with unlimited duration of pulses. The knives were the most frequently used devices, preferably with a length of $5 \mathrm{~mm}$, which enabled us to either cut the tumour or scar tissue precisely slice by slice or to resect by direct coagulation. The use of the blunt probes and forceps was frequently rendered more difficult by detritus covering the instrument du-

Pneumologie 53 (1999) 477-479

(c) Georg Thieme Verlag Stuttgart · New York
N. Schönfeld, T. Temme, M. Serke, R. Loddenkemper

Lungenklinik Heckeshorn, Berlin ring coagulation. Polypes were easily resected with the wire snare, but this kind of tumour was found in four patients only. Major (>100 ml) bleeding occurred in two patients. Obstructing fibrinous membranes were never seen after electrocautery. In conclusion, high-frequency electrocautery is an effective and safe method for endobronchial resection and can be considered a good alternative to the laser as the classical method for endobronchial resection.

Key words: High-frequency electrocautery - Bronchoscopy Stenosis of the airways - Lung cancer

\section{Einleitung}

Höhergradige Stenosen der großen Atemwege bedeuten für den Patienten in der Regel eine beträchtliche Morbidität. Resektionsverfahren bieten sich zur Therapie an, wenn die Stenose überwiegend durch eine endoluminale Verlegung entstanden ist, was am häufigsten im Rahmen von Malignomerkrankungen der Fall ist, seltener durch benigne Tumoren oder bronchoplastische Eingriffe. Neben der altbewährten mechanischen Ausräumung von Gewebe mit der Zange hat sich der Laser in den 80er Jahren zum klassischen thermischen Verfahren entwickelt, wobei das Risiko größerer Blutungen mit etwa $5 \%$ angesetzt werden muß [7].

Obwohl elektrischer Strom zur Resektion endoluminaler Tumoren in der Gastroenterologie bereits seit den 30er Jahren eingesetzt wird, galt die überwiegend als Elektrokauter bezeichnete Technik nach anfänglichen Versuchen lange Zeit als zu gefährlich für den Einsatz im Bronchialsystem [4]. In den 80er Jahren wurde die Methode für den Einsatz über das flexible Fiberbronchoskop weiterentwickelt und damit praktikabel gemacht $[2,5,6]$. Seit kurzem steht nun in Deutschland die Hochfrequenzdiathermie auch kommerziell als technisch ausgereiftes thermisches Verfahren zur Verfügung, das eine Palette von Resektionsinstrumenten bietet, die in Verbindung mit thermischer Energie zur Koagulation angewandt werden können. Wir berichten über die Erfahrungen mit der Technik in unserem Haus aus den letzten drei Jahren.

\section{Patienten und Methodik}

In die retrospektive Studie wurden alle Patienten eingeschlossen, die seit März 1996 im Funktionsbereich Endoskopie der Lungenklinik Heckeshorn entsprechend therapiert wurden. 
Zur Anwendung kam zu Beginn das Hochfrequenzchirurgiegerät HF 120 der Firma Olympus Europe, Hamburg. Es handelte sich um einen neu entwickelten Generator mit einer maximalen Ausgangsleistung von $120 \mathrm{~W}$, der ursprünglich für die Gastroenterologie entwickelt wurde. Später wurde mit dem Olympus-Gerät PSD-20 weitergearbeitet, die beiden Geräte waren in ihren technischen Leistungen vergleichbar. Für unsere Zwecke haben wir aus Sicherheitsgründen stets die monopolare Leistung auf maximal $40 \mathrm{~W}$ bei mittlerem Koagulationseffekt (Schnittmodus) begrenzt.

Die elektrische Energie wurde über verschiedene Instrumente eingebracht, die alle über den Arbeitskanal eines flexiblen Bronchoskops eingeführt werden konnten. Hierbei handelte es sich zunächst um einen Olympus-Prototyp aus der Serie BF 1 T 30 mit spezieller keramischer Isolierung (Bezeichnung A 9908), im weiteren Verlauf konnten aber auch alle neu erworbenen Geräte der Serie BF 1 T 40 zum Einsatz kommen, die standardmäßig entsprechend isoliert waren. Als Instrumente standen die halbkugelförmig geformte Kontaktsonde (Koagulator), eine Zange („Hot Biopsy“-Zange), Vorschneidemesser der Länge 4, 5 und $7 \mathrm{~mm}$ (Papillotomiemesser bzw. Prototypen) sowie Polypektomieschlingen zur Verfügung.

\section{Ergebnisse}

Behandelt wurden 41 Patienten in 58 Sitzungen. Das Patientenkollektiv bestand aus 31 Männern und 10 Frauen, mit einem medianen Altersdurchschnitt von 60 Jahren (Spanne 21 - 80 Jahre) entsprechen. Bei 30 Patienten lag eine maligne Atemwegsstenose vor, bei 8 Patienten bestand eine benigne Anastomosenstenose nach Manschettenresektion oder Lungentransplantation, bei 3 Patienten wurde ein benigner Bronchialtumor diagnostiziert. Die Lokalisation der Stenosen waren: Trachea $n=9$, linker Hauptbronchus $n=20$, rechter Hauptbronchus $n=15$, Zwischenbronchus $n=4$.

Nur 5/58 der Eingriffe wurden in Lokalanästhesie durchgeführt, die anderen in Allgemeinnarkose und Intubation mit dem starren Rohr. Zur Resektion wurden ganz überwiegend die Diathermiemesser verschiedener Länge eingesetzt (49/ 58). Hierfür waren zum einen die günstigen Resektionseigenschaften mit schichtweiser Abtrennung von Tumoranteilen ausschlaggebend, zum anderen wurde bei der Verwendung der Kontaktsonde und der Zange bemerkt, daß die Instrumente rasch durch eine klebrige Detritusschicht bedeckt waren, die vielfache Reinigungen während des Eingriffs erforderlich machten; eine solche Art der Verschmutzung war bei der Benutzung der Messer nur gering zu beobachten. Die Drahtschlingen wurden in nur 4/58 Eingriffen eingesetzt, bedingt durch das seltene Vorkommen gestielter Polypen im Bronchialsystem; wenn die Schlingen zum Einsatz kamen, gelang jedoch stets sehr rasch die Abtrennung auch großer Tumoren $(>1 \mathrm{~cm})$ bzw. großer Tumoranteile.

In 40/58 Eingriffen wurde durch den Einsatz der Hochfrequenzdiathermie eine zufriedenstellende Rekanalisation von mehr als 50\% des zur Verfügung stehenden Lumens erreicht. Wo dies nicht gelang, wurde erst im Verlauf des Eingriffs ein überwiegend extrabronchialer Charakter der Stenose erkennbar oder ein weitergehender Verschluß der Atemwege im Verzweigungsgebiet des rekanalisierten Bronchus aufgedeckt. Es wurden keine technischen Grenzen der Methode erkenn- bar, derentwegen eine geplante Resektion nicht ausgeführt werden konnte. In zwei Fällen kam es zu Blutungen $>100 \mathrm{ml}$, die aber für die Patienten nicht lebensbedrohlich waren und auch außer einer Absaugung keine besonderen Maßnahmen wie eine endobronchiale Tamponade oder eine einseitige Intubation erforderlich machten. Eine Bronchusverlegung durch Fibrinmembranen wurde in keinem einzigen Fall in Zusammenhang mit der Diathermie beobachtet, wobei regelmäßig bronchoskopische Nachkontrollen erfolgten.

Als weitere Therapiemaßnahmen im direkten zeitlichen $\mathrm{Zu}$ sammenhang mit der Hochfrequenzdiathermie wurden in 16 Fällen eine endobronchiale Kleinraumbestrahlung im Afterloadingverfahren durchgeführt, in neun Fällen eine perkutane Strahlentherapie, in drei Fällen eine zytostatische Chemotherapie und in einem Fall eine Operation durchgeführt. Hingewiesen sei noch auf die Rezidivneigung narbiger Stenosen nach Manschettenresektionen, die in einem Fall die Hochfrequenzdiathermie achtmal zum Einsatz kommen ließ, bis sich das Lumen der Anastomose stabilisierte.

\section{Diskussion}

Die von uns gewonnenen Daten über das technisch weiterentwickelte Verfahren der Diathermie zeigen, daß hiermit eine technisch sichere und zuverlässige Alternative zum Laser als dem klassischen Resektionsverfahren zur Verfügung steht. Als einzige relevante Komplikation haben wir in 2/58 Sitzungen eine Blutung von mehr als $100 \mathrm{ml}$ beobachtet, die aber bei beiden Patienten nicht zu einer größeren Bedrohung geführt hat und auch keine endobronchiale Tamponade oder einseitige Intubation erforderlich machte. Allerdings war aus den beiden Ereignissen der Schluß zu ziehen, daß der Koagulationseffekt nicht in jedem Fall ausreichend ist, um eine größere Blutung zu verhindern. Trotz des vermutlich geringeren Blutungsrisikos im Vergleich zum Laser empfehlen wir nach unseren Erfahrungen dieselben Sicherheitsvorkehrungen, wie sie von der Sektion Endoskopie der Deutschen Gesellschaft für Pneumologie für die Lasertherapie angegeben empfohlen werden $[3,8]$. Wir sind dabei dazu übergegangen, Resektionen mittels Hochfrequenzdiathermie von vornherein in Allgemeinnarkose zu planen, was unabhängig von Sicherheitsaspekten den bekannten Vorteil des beruhigten Arbeitsfeldes bietet. Insofern sind wir hier sehr früh von dem bei Einführung der Hochfrequenzdiathermie angestrebten Trend abgerückt, das risikoarme Verfahren möglichst in Lokalanästhesie durchführen zu wollen. Hierzu beigetragen hat allerdings auch - unabhängig von der Hochfrequenzdiathermie die zeitgleiche Etablierung der totalen intravenösen Anästhesie als Standardnarkoseverfahren in unserem Funktionsbereich, das eine bessere Steuerung der Narkose, größere Sicherheit, Bandbreite und Patientenverträglichkeit im Vergleich zu den zuvor durchgeführten Narkosen unter Verwendung der halogenierten Inhalationsanästhetika gewährleistet [1].

Hinsichtlich eventueller Nebenwirkungen war weiterhin zu beobachten, daß sichtbare Begleitschäden der den Tumor umgebenden Bronchuswand praktisch nicht auftraten. Das lag zum einen an der Möglichkeit der sehr präzisen Instrumentenführung, zum anderen aber offenbar auch an der Art der Applikation thermischer Energie. Bei keinem einzigen Patienten haben wir im Rahmen der regelmäßig durchgeführten bronchoskopischen Nachkontrollen eine Verlegung durch 
Fibrinmembranen im Zusammenhang mit dem Diathermieeingriff beobachtet, was ebenfalls auf die Schonung des Gewebes in der Umgebung des Resektionsareals hinweist.

Eine technische Bereicherung ist die Auswahl an Instrumenten, die für die Resektion zur Verfügung stehen. Während Homasson [4] und Sutedja [9] überwiegend mit der Kontaktsonde gearbeitet haben, sind wir praktisch ausschließlich zum Einsatz der Diathermiemesser verschiedener Länge übergegangen. Vorbilder für die verschiedenen Prototypen der Länge 4, 5 und $7 \mathrm{~mm}$ sind Papillotomiemesser aus der gastroenterologischen Endoskopie gewesen. Die Messer erlauben das millimetergenaue Abtrennen von Tumor- oder Narbengewebe, wobei sich der Vorteil dieses präzisen $\mathrm{Ar}$ beitens insbesondere bei der Behandlung von Anastomosenstenosen zeigte, die durch eine Sekundärheilung nach Manschettenresektionen aufgetreten waren. Auch eine direkte Koagulation ist mit den Messern möglich.

Bei der anfänglichen Benutzung der Kontaktsonde und der Diathermiezange war uns aufgefallen, daß sich rasch eine klebrige Detritusschicht um die Instrumente legt, die das weitere Arbeiten behindert und eine wiederholte Reinigung erforderlich macht. Auch dieser Effekt war bei der Benutzung der Diathermiemesser geringer ausgeprägt. Unter Umständen wäre eine solche Verschmutzung der Instrumente mit einer höheren Leistung, d.h. mehr als 40 Watt vermeidbar, jedoch haben wir zu einer solchen möglicherweise risikoreichen Maßnahme in Anbetracht des guten Resektionserfolges mittels der Messer keine Veranlassung gesehen.

In wenigen Fällen war die Abtragung gestielter polypöser Tumoren mit der Schlinge möglich und dann sehr schnell zu bewerkstelligen. Ansonsten dürfte nach unserem Eindruck der einzige Vorteil des Lasers gegenüber der Diathermie noch darin bestehen, daß man mit der koagulierenden Abtragung schneller vorankommt, wobei es hierzu jedoch keine vergleichenden Daten gibt. Insgesamt ist die Hochfrequenzdiathermie ein sicheres und präzises Verfahren in der Behandlung maligner und benigner Atemwegsstenosen und stellt eine kostengünstigere und wahrscheinlich auch risikoärmere Alternative zum Laser dar.

Wir danken insbesondere Herrn Yoshio Tashiro, aber auch anderen Mitarbeitern der Firma Olympus Optical, Hamburg, für die technische Unterstützung. Weiterhin wurde die Arbeit unterstützt durch den Verein zur Förderung der Pneumologie und Thoraxchirurgie in Berlin-Heckeshorn e.V.

\section{Literatur}

${ }^{1}$ Coates D. "Diprifusor" for general and day-case surgery. Anasthesia 1998; 53, suppl 1: $46-48$

2 Cunningham L, Wendell G, Berkowitz L, Schulman ES, Promisloff R. Treatment of tracheobronchial granular cell myoblastomas with endoscopic bipolar cautery. Chest 1989; 96: 427 - 429

${ }^{3}$ Deutsche Gesellschaft für Pneumologie, Arbeitsgruppe „Qualitätssicherung in der Bronchologie“. Empfehlungen zur bronchoskopischen Behandlung tracheobronchialer Verschlüsse, Stenosen und muraler maligner Tumoren. Pneumologie 1998; 52: 243 248

${ }^{4}$ Homasson JP. Endobronchial electrocautery. Sem Respir Crit Care Med 1997; 18: 535 - 543
${ }^{5}$ Hooper RG, Jackson FN. Endobronchial electrocautery. Chest 1988; 96: $595-598$

${ }^{6}$ Marsh BR. Bipolar cautery for the fiberoptic bronchoscope. Ann Otol Laryngol 1987; 96: 120 - 121

${ }^{7}$ Ramser ER, Beamis JF. Laser bronchoscopy. Clin Chest Med 1995; 16: $415-426$

${ }^{8}$ Schönfeld N, Loddenkemper R. Interventionelle bronchoskopische Verfahren: Durchführung. Dtsch Med Wschr 1999; 124: $697-699$

${ }^{9}$ Sutedja G, Kralingen van K, Schramel FMNH, Postmus PE. Fibreoptic bronchoscopic electrosurgery under local anaesthesia for rapid palliation in patients with central airway malignancies: a preliminary report. Thorax 1994; 94: 1243 - 1246

\section{Dr. Nicolas Schönfeld}

Pneumologische Abteilung II

Lungenklinik Heckeshorn

Zum Heckeshorn 33

14109 Berlin

E-mail: loddheck@zedat.fu-berlin.de 